\title{
For everything a season? A month-by-month analysis of social network resources in later life
}

\author{
Laura Upenieks, Jason Settels, Markus H. Schafer
}

\begin{abstract}
:
It is widely acknowledged that informal social ties provide older persons with many resources that serve to protect and improve their levels of health and well-being. Most studies on this topic, however, ignore the month or season of the year during which data was accumulated. This study proposes two hypotheses to explain seniors' social network resources over the calendar year: the "fluctuation hypothesis", which proposes that seasonal variation, in the form of weather fluctuations, institutional calendars, and holidays, might influence the social lives and resources of older persons, and the "network stability" perspective, which, informed by tenets of convoy theory and socioemotional selectivity theory, emphasizes the increasing importance of close network ties as individuals age and the stability of these ties. Using two waves (2005-2006 and 2010-2011) of the National Social Life, Health, and Aging Project (NSHAP), a nationally representative sample of community-dwelling older adults aged 57-85 in the United States, we examine a diverse set of nine social connectedness outcomes. Results, overall, support the network stability perspective, as the only social connectedness outcome found to significantly vary by month of year was average closeness with network members. We conclude by suggesting some methodological considerations for survey research and by noting how these findings complement the growing literature on inter-year fluctuation in social networks and social support. Changes in older adults' networks, while frequently observable over the course of years, do not seem to be seasonally patterned.

https://doi.org/10.1016/j.ssresearch.2017.09.004
\end{abstract}

https://www.sciencedirect.com/science/article/abs/pii/S0049089X16307608 